\title{
Curved Space: Theory of Everything
}

S. Chen Rheast LLC, 1331 Lamar St, Houston, TX 77010, USA. chen@rheast.com

\begin{abstract}
Matter and energy are both converted from curved space, they are different forms of the same thing. The flow of space creates the gravitation, and the increase of space causes the expansion of the universe. Matter curves in two different directions of one dimension creates two types of electric charges: positive and negative. Matter curves in three different dimensions creates three values or charges of quark's color: red, green, and blue. The equivalent equation of space: $S=E \mathrm{c}^{2}=m \mathrm{c}^{4}$. The gravitation of hollow sphere space: $S_{\mu \nu}=4 \pi G m=(4 / 3) \pi\left((r+a)^{3}-r^{3}\right)$.
\end{abstract}

\section{Introduction}

At the death of a star, it will undergo a contraction and blows off its outer envelope to form a planetary nebula [6,7]. If the star collapses to within its Schwarzschild radius, it forms a black hole ${ }^{[8]}$. According to the Newton's law of universal gravitation ${ }^{[1]}$, the equation for universal gravitation is:

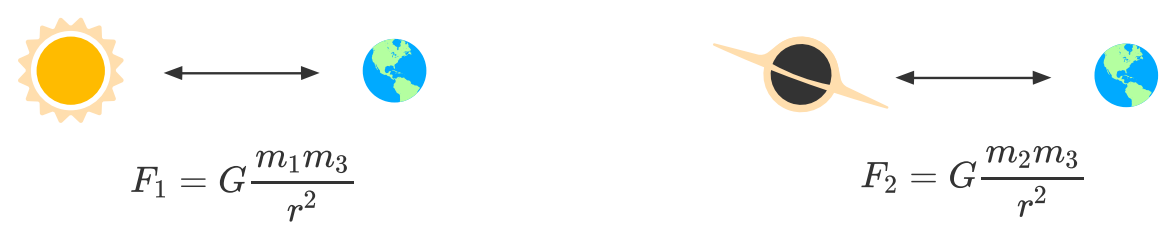

Figure 1. Star forms a black hole

Where $F_{1}$ is the gravitation between the planet and the star before its collapses, $F_{2}$ is the gravitation between the planet and the black hole after the star collapses, $m_{1}$ is the masses of the star, $m_{2}$ is the masses of the black hole, $m_{3}$ is the masses of the planet, $r$ is the distance between the centers of their masses, and $G$ is the constant of gravitation. The gravitation of the black hole is extremely strong, and nothing can escape from it even the light ${ }^{[9]}$, so the gravitation $F_{2}$ is bigger than $F_{1}$. The star blows off its outer envelope and lost mass when it collapses into a black hole ${ }^{[10]}$, assuming the star loses $2 \%$ of its mass during this period, so the mass $m_{2}$ is $98 \%$ of $m_{1}$. The form can be rewritten to:

$$
F_{1}=G \frac{m_{1} m_{3}}{r^{2}} \quad<\quad F_{2}=G \frac{0.98 m_{1} m_{3}}{r^{2}}
$$

After removing the same parameters, the form reduces to:

$1<0.98$

How could it be possible that 1 less than 0.98? The mass $m_{1}$ decrease, and the distance $r$ have not changed, which means the Newton's constant of gravitation $G$ seems to be increased.

In Einstein's theory of relativity, matter curves spacetime, the Einstein field equations can be written in the form [4]:

$$
G_{\mu \nu}=R_{\mu \nu}-\frac{1}{2} g_{\mu \nu} R=\frac{8 \pi G}{c^{4}} T_{\mu \nu}
$$

Where $G_{\mu \nu}$ is the Einstein tensor and $G$ is the Newton's constant of gravitation. But if the Newton's constant of gravitation $G$ is constantly changing, does Einstein's theory still be correct?

\section{Model}

What if the fact is reversed that matter and energy are both converted from curved space?

Matter releases energy through nannihilation, fission and fusion ${ }^{[2,3]}$ because matter is converted from energy, they are different forms of the same thing. So matter curves spacetime because matter is converted from curved space, the flow of space released 
by matter creates gravitation. The greater the amount of space released, the faster the speed of space flow, and the greater the gravitation generated. If the space released by one object is much larger than the other one, the flow of space will narrows the distance between two objects. It is space that moves, not objects.

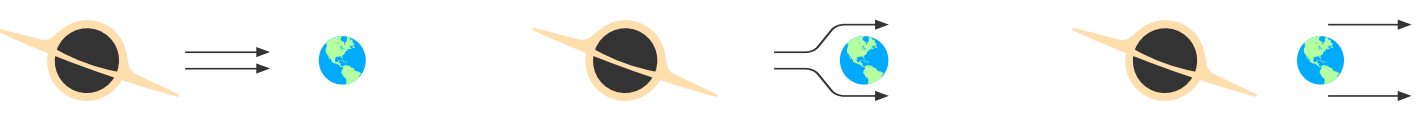

Figure 2. Gravitation

When two galaxies are very far apart, the space they released accumulates in the middle and extends their distance, so the expansion of the universe and the phenomenon of redshift ${ }^{[11]}$ are caused by the increase of space.

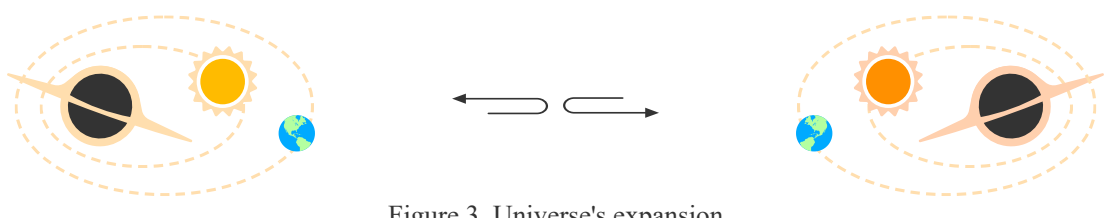

Figure 3. Universe's expansion

Therefore, the space curves in one direction of one dimension create matter and energy.

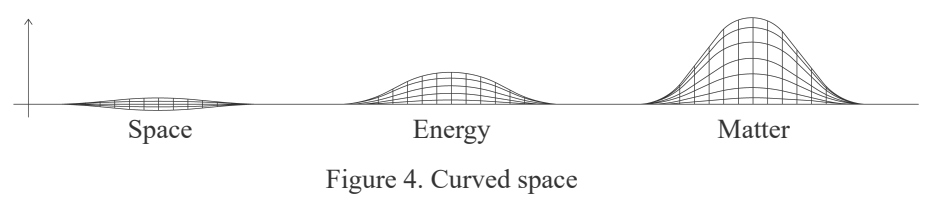

Since the electron is matter, and one dimension has only two directions, so matter curves in two different directions of one dimension create two types of the electric charges: positive and negative. Like charges repel each other because they occupied the same position of one dimension, and unlike charges attract each other because they occupied the opposite position of one dimension. Since they are all matter, so they will only attract or repel each other, not annihilation.
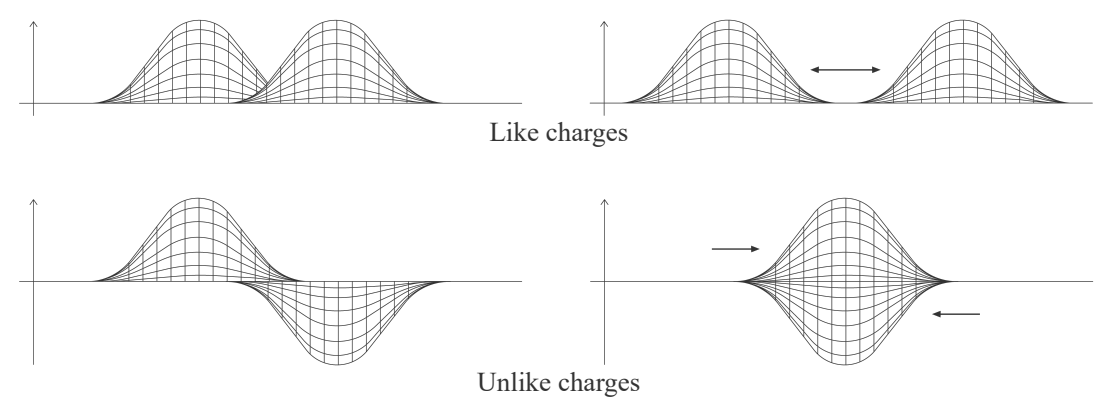

Figure 5. Electric charges

Quark and antiquark also follow the same pattern: matter curves in three different dimensions creates three values or charges of quark's color: red, green, and blue ${ }^{[12]}$. The experimental discovery of neutrino oscillation ${ }^{[13,14,15]}$ proves that those three values of quark's color can be converted into each other: they are different forms of the same thing.
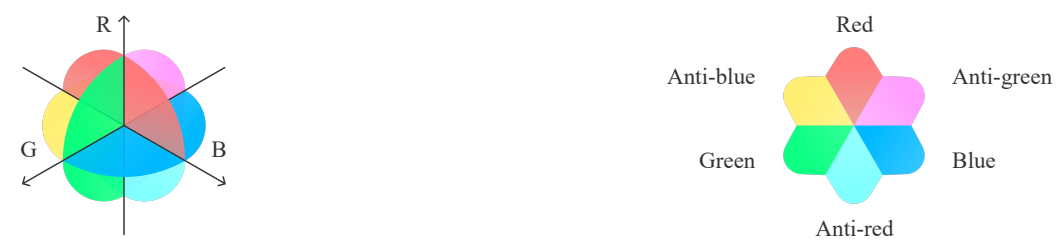

Figure 6. Quark's color

Since there are only three values of quark's color, so the space has only three dimensions. Therefore, the space curves in six different directions of three dimensions creates six types of matter: ordinary matter, antimatter, two types of dark matter, and two types of anti-dark matter. Matter and antimatter in the opposite directions will attract and annihilate each other. Matter and dark matter which perpendicular to each other in other dimension have no electromagnetic force. Matter, antimatter, dark matter and anti-dark matter are both affected by gravitation. When the Big Bang occurred, matter and antimatter moved in two opposite 
directions, so antimatter was on the other side of the universe, and there are only dark matter and anti-dark matter in the current observable universe. The particle collision experiment ${ }^{[16]}$ proves that ordinary matter can be converted into antimatter: they are different forms of the same thing.

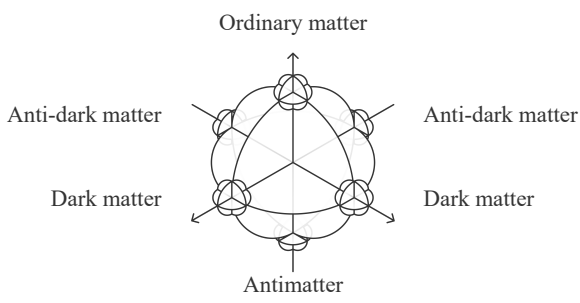

Figure 7. Six types of matter

Matter converted from curved space, and matter curves time. In double-slit experiment, an interference pattern emerges when the particles are allowed to build up one by one ${ }^{[17]}$. The particle interferes with itself because matter curves time, and the particle of the past interfere with the particle of the future. In which-way experiments, if particle detectors are positioned at the slits, showing through which slit a photon goes, the interference pattern will disappear ${ }^{[18]}$. The mass of the observer is much bigger than the particle, when the observation behavior occurs, the time of the observer swallowed the time of the particle, just like the black hole swallowing the star, which causes the wave function collapse. The Wheeler's delayed choice experiments demonstrate that extracting "which path" information after a particle passes through the slits can seem to retroactively alter its previous behavior at the slits ${ }^{[19]}$. Matter curves time, and the particle of the future interfere with the particle of the past, so the current behavior had an impact in the past. The quantum eraser experiments demonstrate that wave behavior can be restored by erasing or otherwise making permanently unavailable the "which path" information ${ }^{[20]}$. Since the time of the particle has no connection with the observer, no wave function collapse occurs.

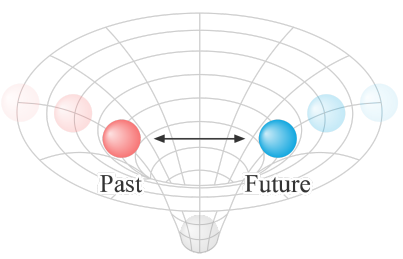

Figure 8. Matter curves time

Every elementary particle is matter, and they are all converted from curved space, so they all have rest mass, but current commonly accepted physical theories imply or assume the photon to be strictly massless.

The relativistic expressions for $E$ and $p$ obey the relativistic energy-momentum relation [21]:

$$
E^{2}=(p c)^{2}+\left(m_{0} c^{2}\right)^{2}
$$

Where the $m_{0}$ is the rest mass, or the invariant mass for systems, and $E$ is the total energy. Since the Standard Model of particle physics defines the rest mass of the photon is zero ${ }^{[22]}$, so the form reduces to:

$$
m_{0}=0 \quad E=p c
$$

Since the photon obeys the Newton's second law ${ }^{[1]}$, so its momentum is:

$$
p=m v
$$

And the Lorentz factor $\gamma$ is defined as ${ }^{[23]}$ :

$$
\gamma=\frac{1}{\sqrt{1-(v / c)^{2}}}
$$

$$
p=m v=m_{0} v \gamma=\frac{m_{0} v}{\sqrt{1-(v / c)^{2}}}
$$

Since the Standard Model of particle physics defines the rest mass of the photon is zero ${ }^{[22]}$, so the form reduces to:

$$
m_{0}=0 \quad p=m_{0} v \gamma=0
$$


Obviously, the rest mass of the photon cannot be zero, which causes the momentum and the relativistic mass become zero. In Newton's law of universal gravitation, the gravitational acceleration is [1]:

$$
a=\frac{G m_{0} / r^{2}}{\sqrt{1-(v / c)^{2}}}
$$

In Einstein's theory of relativity, the stress-energy $T^{\alpha \beta}$ of a non-interacting particle with rest mass $m_{0}$ and trajectory $\mathrm{X}_{\mathrm{p}}(t)$ is $[2,3]$ :

$$
T^{\alpha \beta}(\mathbf{x}, t)=\frac{m_{0} v^{\alpha}(t) v^{\beta}(t)}{\sqrt{1-(v / c)^{2}}} \delta\left(\mathbf{x}-\mathbf{x}_{\mathrm{p}}(t)\right)
$$

If the photon has no rest mass, it will not be affected by the gravitation, and light can escape from the black hole, which is inconsistent with the observations ${ }^{[9]}$.

Of course, there is no doubt that the Standard Model of particle physics is $100 \%$ accurate, so the blame is on the photon. The one that made the mistake must be the photon, it should run exactly as expected by the scientists.

By the way, the Standard Model also defines the rest mass of the neutrino is zero [22]. Unfortunately, the experimental discovery of neutrino oscillation, and thus neutrino mass, by the Super-Kamiokande Observatory and the Sudbury Neutrino Observatories, was found that the neutrino has a non-zero rest mass ${ }^{[13,14,15]}$.

\section{Equivalent equation of space}

In Einstein's theory of relativity, matter curves spacetime, the Einstein field equations can be written in the form ${ }^{[2,3,4]}$ :

$$
G_{\mu \nu}=R_{\mu \nu}-\frac{1}{2} g_{\mu \nu} R=\frac{8 \pi G}{c^{4}} T_{\mu \nu} \quad \kappa=\frac{8 \pi G}{c^{4}}
$$

Where $G_{\mu v}$ is the Einstein tensor, $T_{\mu v}$ is the stress-energy tensor, $G$ is the Newton's constant of gravitation, c is the speed of light in vacuum, and $\kappa$ is the Einstein constant of gravitation. Since the Newton's law of universal gravitation doesn't apply to the black hole, so use the geometrized unit system $4 \pi G=1$ to remove the Newton's constant of gravitation to avoid mistake. The form can be rewritten to:

$$
4 \pi G=1 \quad \kappa=\frac{2}{c^{4}}
$$

Since the fact is reversed that matter and energy are both converted from curved space, so the form should also be reversed, and the space $S$ is proportional to the mass $m$ and the fourth power of speed of light $\mathrm{c}^{4}$. Since matter converted from energy, and energy converted from curved space, the equivalent equation of space $S$ thus takes the form:

$$
E=m c^{2} \quad S=E c^{2}=m c^{4}
$$

The intensity of gravitation depends on how much space is released, the black hole loses mass but increase gravitation proves that even if the mass of the object decrease, gravitation can still increase. The gravitation will not increase for no reason, there is certainly a corresponding cost: loss of mass or energy. However, the mass loses is extremely small when matter released space, one kilogram of matter equals to $8.07760871 \times 10^{33} \mathrm{~m}^{4} / \mathrm{s}^{4}$ of space, which is difficult to be observed, and the energy also involved in this process.

\section{Gravitation of hollow sphere space}

Since there are only three values of quark's color, so the space has only three dimensions. Therefore, the space that released by the matter is a three-dimensional sphere. When the space flows outward, it forms a hollow sphere, its volume can be written in the form: 


$$
V_{3}=\frac{4}{3} \pi r^{3}
$$

$$
S_{\mu \nu}=\frac{4}{3} \pi\left((r+a)^{3}-r^{3}\right)
$$

Where $S_{\mu \nu}$ is the space that released by the matter, $r$ is the radius of the sphere, and $a$ is the gravitational acceleration. When the space flows outward, its volume remains unchanged, and the gravitational acceleration $a_{1}$ of one distance can be directly used to obtain the gravitational acceleration $a_{2}$ of another distance. The form reduces to:

$$
S_{\mu \nu}=\frac{4}{3} \pi\left(\left(r_{1}+a_{1}\right)^{3}-r_{1}^{3}\right)=\frac{4}{3} \pi\left(\left(r_{2}+a_{2}\right)^{3}-r_{2}{ }^{3}\right) \quad a_{2}=\sqrt[3]{\left(r_{1}+a_{1}\right)^{3}-r_{1}{ }^{3}+r_{2}{ }^{3}}-r_{2}
$$

In Newton's law of universal gravitation, the gravitational acceleration is [1]:

$$
a=G \frac{m}{r^{2}}
$$

The mass of Earth is about $5.97237 \times 10^{24} \mathrm{~kg}$, and the average distance from center to surface is about $6.37123 \times 10^{6} \mathrm{~m}$ [24,25]. In Newton's law of universal gravitation, the value of the Newton's constant of gravitation is about $6.67408 \times 10^{-11} \mathrm{~m}^{3} \cdot \mathrm{kg}^{-1} \cdot \mathrm{s}^{-2}[26]$, and the gravitational acceleration $a_{1}$ of the Earth's surface is:

$$
a_{1}=G \frac{m}{r_{1}^{2}} \approx 9.8195491164 \mathrm{~m} / \mathrm{s}^{2}
$$

If the distance up to $10^{7} \mathrm{~m}$, the gravitational acceleration $a_{2}$ is:

$$
a_{2}=G \frac{m}{r_{2}{ }^{2}} \approx 3.9860075169 \mathrm{~m} / \mathrm{s}^{2}
$$

When the gravitational acceleration $a_{1}$ of the hollow sphere space is equals to the Newton's gravitational acceleration $a_{1}$ at the same distance, the gravitational acceleration $a_{2}$ of hollow sphere space is:

$$
a_{2}=\sqrt[3]{\left(r_{1}+a_{1}\right)^{3}-r_{1}{ }^{3}+r_{2}{ }^{3}}-r_{2} \approx 3.9860120713 \mathrm{~m} / \mathrm{s}^{2}
$$

As you can see, these two values of the gravitational acceleration $a_{2}$ are extremely close, which proves that the gravitation of hollow sphere space can be used to calculate the gravitational acceleration. But when the distance is very far away from Earth, please use a professional software to calculate the cube root, otherwise the calculator will return zero or a negative value.

In different distances, the gravitational acceleration of Earth in two types of models is shown in the following table:

\begin{tabular}{|c|c|c|c|c|c|c|c|}
\hline Distance of Earth & $0 \mathrm{~m}$ & $1.15 \times 10^{3} \mathrm{~m}$ & $10^{4} \mathrm{~m}$ & $10^{5} \mathrm{~m}$ & $10^{6} \mathrm{~m}$ & $10^{7} \mathrm{~m}$ & $10^{8} \mathrm{~m}$ \\
\hline $\begin{array}{c}\text { Newton's law of } \\
\text { universal gravitation }\end{array}$ & $\infty$ & $3.01 \times 10^{8} \mathrm{~m} / \mathrm{s}^{2}$ & $3986007 \mathrm{~m} / \mathrm{s}^{2}$ & $39860 \mathrm{~m} / \mathrm{s}^{2}$ & $398.600 \mathrm{~m} / \mathrm{s}^{2}$ & $3.98600751 \mathrm{~m} / \mathrm{s}^{2}$ & $0.03986007 \mathrm{~m} / \mathrm{s}^{2}$ \\
\hline $\begin{array}{c}\text { Gravitation of } \\
\text { hollow sphere space }\end{array}$ & $106141 \mathrm{~m} / \mathrm{s}^{2}$ & $104991 \mathrm{~m} / \mathrm{s}^{2}$ & $96171 \mathrm{~m} / \mathrm{s}^{2}$ & $29976 \mathrm{~m} / \mathrm{s}^{2}$ & $398.442 \mathrm{~m} / \mathrm{s}^{2}$ & $3.98601207 \mathrm{~m} / \mathrm{s}^{2}$ & $0.03986012 \mathrm{~m} / \mathrm{s}^{2}$ \\
\hline
\end{tabular}

Table 1. Gravitational acceleration of Earth

When the distance is close to zero, Newton's gravitational acceleration is infinite, which is obviously wrong. The gravitation of hollow sphere space is more accurate, and it doesn't required the Newton's constant of gravitation.

The Schwarzschild radius ${ }^{[8]}$ is a physical parameter that appears in the Schwarzschild solution to Einstein's field equations [5], corresponding to the radius defining the event horizon of a Schwarzschild black hole, which can be written in the form:

$$
r_{s}=\frac{2 G m}{c^{2}}
$$

The Schwarzschild radius of Earth is about $0.00887 \mathrm{~m}$, but in Newton's gravitation, when the distance reaches about $1150 \mathrm{~m}$, 
the gravitational acceleration is about $3.01 \times 10^{8} \mathrm{~m} / \mathrm{s}^{2}$, it is bigger than the speed of light in vacuum approximately $2.9979 \times 10^{8} \mathrm{~m} / \mathrm{s}^{2}$, and it forms a black hole, which is obviously wrong.

The mass of Moon is about $7.342 \times 10^{22} \mathrm{~kg}$, its mean radius is about $1.737 \times 10^{6} \mathrm{~m}$, its surface is about $1.624 \mathrm{~m} / \mathrm{s}^{2}$, and the timeaveraged distance between Earth and Moon centers is about $3.844 \times 10^{8} \mathrm{~m}[27,28,29]$.

In different distances, the gravitational acceleration of Moon in two types of models is shown in the following table:

\begin{tabular}{|c|c|c|c|c|c|c|c|}
\hline Distance of Moon & $0 \mathrm{~m}$ & $1.27 \times 10^{2} \mathrm{~m}$ & $10^{5} \mathrm{~m}$ & $10^{6} \mathrm{~m}$ & $10^{7} \mathrm{~m}$ & $10^{8} \mathrm{~m}$ & $3.844 \times 10^{8} \mathrm{~m}$ \\
\hline $\begin{array}{c}\text { Newton's law of } \\
\text { universal gravitation }\end{array}$ & $\infty$ & $3.03 \times 10^{8} \mathrm{~m} / \mathrm{s}^{2}$ & $490.01 \mathrm{~m} / \mathrm{s}^{2}$ & $4.90010953 \mathrm{~m} / \mathrm{s}^{2}$ & $0.04900109 \mathrm{~m} / \mathrm{s}^{2}$ & $0.00049001 \mathrm{~m} / \mathrm{s}^{2}$ & $0.00003316 \mathrm{~m} / \mathrm{s}^{2}$ \\
\hline $\begin{array}{c}\text { Gravitation of } \\
\text { hollow sphere space }\end{array}$ & $24496 \mathrm{~m} / \mathrm{s}^{2}$ & $24369 \mathrm{~m} / \mathrm{s}^{2}$ & $487.60 \mathrm{~m} / \mathrm{s}^{2}$ & $4.89986302 \mathrm{~m} / \mathrm{s}^{2}$ & $0.04899887 \mathrm{~m} / \mathrm{s}^{2}$ & $0.00048998 \mathrm{~m} / \mathrm{s}^{2}$ & $0.00003316 \mathrm{~m} / \mathrm{s}^{2}$ \\
\hline
\end{tabular}

Table 2. Gravitational acceleration of Moon

The mass of Sun is about $1.9885 \times 10^{30} \mathrm{~kg}$, its mean radius is about $6.96342 \times 10^{8} \mathrm{~m}$, its surface is about $273.698 \mathrm{~m} / \mathrm{s}^{2}$, and the mean distance between Earth and Sun centers is about $1.496 \times 10^{11} \mathrm{~m}^{[30,31]}$.

In different distances, the gravitational acceleration of Sun in two types of models is shown in the following table:

\begin{tabular}{|c|c|c|c|c|c|c|c|}
\hline Distance of Sun & $0 \mathrm{~m}$ & $6.65 \times 10^{5} \mathrm{~m}$ & $10^{7} \mathrm{~m}$ & $10^{9} \mathrm{~m}$ & $10^{10} \mathrm{~m}$ & $10^{11} \mathrm{~m}$ & $1.496 \times 10^{11} \mathrm{~m}$ \\
\hline $\begin{array}{c}\text { Newton's law of } \\
\text { universal gravitation }\end{array}$ & $\infty$ & $3.001 \times 10^{8} \mathrm{~m} / \mathrm{s}^{2}$ & $1327140 \mathrm{~m} / \mathrm{s}^{2}$ & $132.714 \mathrm{~m} / \mathrm{s}^{2}$ & $1.32714 \mathrm{~m} / \mathrm{s}^{2}$ & $0.01327140 \mathrm{~m} / \mathrm{s}^{2}$ & $0.00592998 \mathrm{~m} / \mathrm{s}^{2}$ \\
\hline $\begin{array}{c}\text { Gravitation of } \\
\text { hollow sphere space }\end{array}$ & $7356638 \mathrm{~m} / \mathrm{s}^{2}$ & $6693449 \mathrm{~m} / \mathrm{s}^{2}$ & $1181938 \mathrm{~m} / \mathrm{s}^{2}$ & $132.714 \mathrm{~m} / \mathrm{s}^{2}$ & $1.32714 \mathrm{~m} / \mathrm{s}^{2}$ & $0.01327140 \mathrm{~m} / \mathrm{s}^{2}$ & $0.00592998 \mathrm{~m} / \mathrm{s}^{2}$ \\
\hline
\end{tabular}

Table 3. Gravitational acceleration of Sun

Why are the values of the two formulas extremely close at the long distance? Expanding the formula, the gravitation of hollow sphere space is:

$$
S_{\mu \nu}=\frac{4}{3} \pi\left((a+r)^{3}-r^{3}\right)=\frac{4}{3} \pi\left(3 r^{2} a+3 r a^{2}+a^{3}\right)=4 \pi r^{2} a\left(1+\frac{a}{r}+\frac{a^{2}}{3 r^{2}}\right)
$$

Then use a new variable $\beta$ to represent $1+a / r+a^{2} / 3 r^{2}$, the form reduces to:

$$
S_{\mu \nu}=4 \pi r^{2} a \beta \quad \beta=1+\frac{a}{r}+\frac{a^{2}}{3 r^{2}} \quad \quad \lim _{r \rightarrow \infty} \beta=1
$$

The expression means that $\beta$ can be made to be as close to 1 as desired, by making $r$ sufficiently close to infinity. On the surface of Earth, the value of gravitational acceleration $a$ divided by the distance $r$ is about 0.00000153 , and it is extremely small, which can be omitted. The form can be rewritten to:

$$
S_{\mu \nu}=4 \pi r^{2} a \quad \beta=1
$$

Since the acceleration of the two formulas are equal at the long distance, the form reduces to:

$$
a=\frac{S_{\mu \nu}}{4 \pi r^{2}}=\frac{G m}{r^{2}}
$$

After removing the same parameters, the gravitation of hollow sphere space $S_{\mu v}$ thus takes the form:

$$
S_{\mu \nu}=4 \pi G m
$$

The result shows that the space released by matter per kilogram is exactly equal to $4 \pi G$, which proves that matter is converted from curved space: they are different forms of the same thing. Compared with Newton's law of universal gravitation, there is only one more variable $\beta$ in the gravitation of hollow sphere space, and it is extremely close to 1 at the long distance. 


$$
S_{\mu \nu}=4 \pi G m=4 \pi r^{2} a \beta
$$

$$
a=\frac{4 \pi G m}{4 \pi r^{2} \beta}=\frac{G m}{r^{2}} \frac{1}{\beta}
$$

The further the distance between the objects, the closer the values of the formulas.

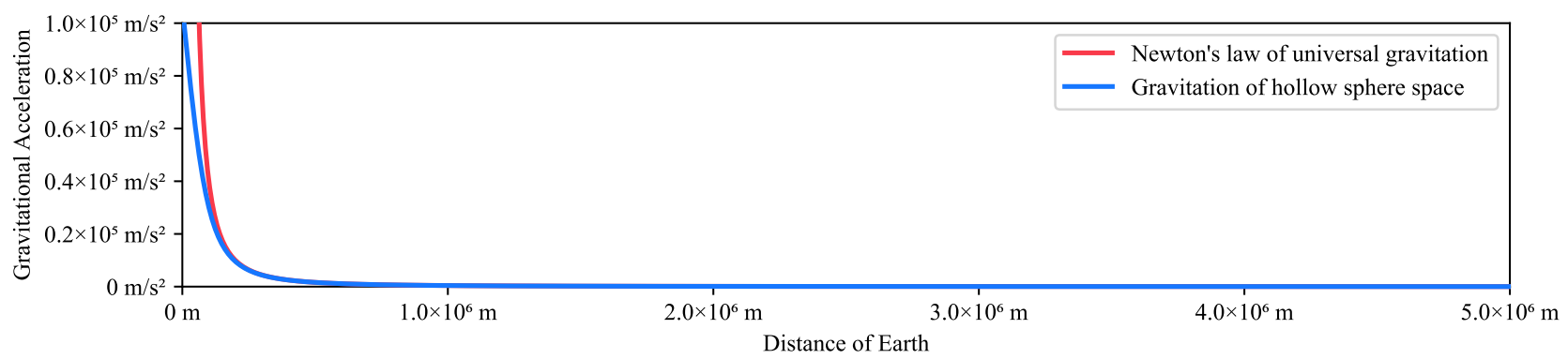

Figure 9. Gravitational acceleration of Earth

The relationship between the gravitation of hollow sphere space and the speed of light is:

$$
S=E c^{2}=m c^{4}
$$

$$
S_{\mu \nu}=\frac{4 \pi G}{c^{4}} S
$$

Of course, under ordinary circumstances, if the Newton's "constant" of gravitation $G$ doesn't change too much, it can still be used to calculate the gravitational acceleration, but the formula needs to be modified to avoid an infinity value when the distance is close to zero. The form can be rewritten to:

$$
S_{\mu \nu}=4 \pi G m=\frac{4}{3} \pi\left((r+a)^{3}-r^{3}\right) \quad a=\sqrt[3]{3 G m+r^{3}}-r
$$

On the surface of Earth, the gravitational acceleration of the modified version of the Newton's gravitation is:

$$
a=\sqrt[3]{3 G m+r^{3}}-r \approx 9.8195339692 m / s^{2}
$$

As you can see, this value is still extremely close to the original. And most importantly, this formula doesn't add new variables.

\section{Discussion}

As of this writing, graviton has not been found yet.

\section{Funding}

This research did not receive any specific grant from funding agencies in the public, commercial, or not-for-profit sectors.

\section{References}

1. I. Newton. (1687). "Philosophiae Naturalis Principia Mathematica". The Mathematical Principles of Natural Philosophy.

2. A. Einstein. (1905). "Ist die Trägheit eines Körpers von seinem Energieinhalt abhängig". Annalen der Physik, 4, 18, 639-641.

3. A. Einstein, M. Grossmann. (1913). "Entwurf einer verallgemeinerten Relativitätstheorie und eine Theorie der Gravitation". Zeitschrift für Mathematik und Physik, 62, 225-244, 245-261.

4. A. Einstein, A.D. Fokker. (1913). "Nordströmsche Gravitationstheorie vom Standpunkt des absoluten Differentialkalküls". Annalen der Physik, 4, 40, 551-560.

5. A. Einstein. (1916). "Die Grundlage der allgemeinen Relativitätstheorie". Annalen der Physik, Vierte Folge, Band 49, 769-822.

6. R. Penrose. (1965). "Gravitational Collapse and Space-Time Singularities". Physical Review Letters. American Physical Society, 14, 57-59. doi: $10.1103 /$ physrevlett.14.57

7. A. Frankowski, N. Soker. (2009). "Very late thermal pulses influenced by accretion in planetary nebulae". New Astronomy, 14, 8, 
654-658. doi: 10.1016/j.newast.2009.03.006

8. K. Schwarzschild. (1916). "Über das Gravitationsfeld eines Massenpunktes nach der Einsteinschen Theorie". Sitzungsberichte der Königlich Preußischen Akademie der Wissenschaften, Seite 189-196.

9. D. Clery. (2020). "Black holes caught in the act of swallowing stars". Science, 367, 6477, 495. doi: $\underline{10.1126 / \text { science.367.6477.495 }}$

10. S.W. Hawking. (1974). "Black hole explosions". Nature 248, 30-31. doi: 10.1038/248030a0

11. E. Hubble. (1929). "A relation between distance and radial velocity among extra-galactic nebulae". PNAS 15, 3, 168-173. doi: $\underline{10}$. 1073/pnas.15.3.168

12. O.W. Greenberg. (1964). "Spin and Unitary Spin Independence in a Paraquark Model of Baryons and Mesons". Physical Review Letters, 13, 598-602.

13. B. Pontecorvo. (1958). "Mesonium and Antimesonium". Soviet Journal of Experimental and Theoretical Physics, 6, $429-431$.

14. E. Kearns, T. Kajita, Y. Totsuka. (1999). "Detecting Massive Neutrinos". Scientific American, 281, 64-71. doi: 10.1038/scientificam erican0899-64

15. A. McDonald, J. Klein, D. Wark. (2006). "Solving the Solar Neutrino Problem". Scientific American, 288, 40-49. doi: 10.1038/scien tificamerican0403-40

16. V. Hatton. (1991). "Operational history of the SPS collider 1981-1990". Conference Record of the 1991 IEEE Particle Accelerator Conference, 2952-2954. doi: 10.1109/PAC.1991.165151

17. R. Pfleegor, L. Mandel. (1967). "Interference of Independent Photon Beams". Physical Review, 159, 5, 1084-1088. doi: 10.1103/P hysRev.159.1084

18. W. Wootters, W. Zurek. (1979). "Complementarity in the double-slit experiment: Quantum nonseparability and a quantitative statement of Bohr's principle". Physical Review D, 19, 2, 473-484. doi: 10.1103/PhysRevD.19.473

19. A. Peruzzo, P. Shadbolt, et al. (2012). "A Quantum Delayed-Choice Experiment". Science, 338, 6107, 634-637. doi: 10.1126/scien ce.1226719

20. X. Ma, J. Kofler, et al. (2012). "Quantum erasure with causally disconnected choice". Proceedings of the National Academy of Sciences, 110, 4, 110-1226. doi: 10.1073/pnas.1213201110

21. M. Alonso, E. Finn. (1968). "Fundamental University Physics Volume III: Quantum and Statistical Physics". Addison Wesley, ISBN 978-0-201-00262-1.

22. R. Oerter. (2006). "The Theory of Almost Everything: The Standard Model, the Unsung Triumph of Modern Physics". Penguin Group, ISBN 978-0-13-236678-6.

23. J. Forshaw, G. Smith. (2014). "Dynamics and Relativity". John Wiley \& Sons, ISBN 978-1-118-93329-9.

24. B. Luzum, N. Capitaine, A. Fienga, et al. (2011). "The IAU 2009 system of astronomical constants: The report of the IAU working group on numerical standards for Fundamental Astronomy". Celestial Mechanics and Dynamical Astronomy, 110, $293-304$. doi: $10.1007 /$ s10569-011-9352-4

25. F. Chambat, B. Valette. (2001). "Mean radius, mass, and inertia for reference Earth models". Physics of the Earth and Planetary Interiors, 124, 3-4, 234-253. doi: 10.1016/S0031-9201(01) 00200-X

26. P. Mohr, D. Newell, B. Taylor. (2016). "CODATA Recommended Values of the Fundamental Physical Constants: 2014". Journal of Physical and Chemical Reference Data, 45, 4, 1527-1605. doi: 10.1063/1.4954402

27. M. Wieczorek, B. Jolliff, A. Khan, et al. (2006). "The Constitution and Structure of the Lunar Interior". Reviews in Mineralogy and Geochemistry, 60, 1, 221-364. doi: 10.2138/rmg.2006.60.3

28. C. Hirt, W.E. Featherstone. (2012). "A 1.5 km-resolution field model of the Moon". Earth and Planetary Science Letters, 329-330, 22-30. doi: 10.1016/j.epsl.2012.02.012

29. J. Battat, T. Murphy, E. Adelberger, et al. (2009). "The Apache Point Observatory Lunar Laser-ranging Operation (APOLLO): Two Years of Millimeter-Precision Measurements of the Earth-Moon Range". Astronomical Society of the Pacific, 121, 875, 29-40. doi: $\underline{10.1086 / 596748}$

30. G. Poole. (2019). "Cosmic Power Generation and Gravity". Journal of High Energy Physics, Gravitation and Cosmology, 5, 920927. doi: 10.4236/jhepgc.2019.53047

31. E. Pitjeva, E. Standish. (2009). "Proposals for the masses of the three largest asteroids, the Moon-Earth mass ratio and the Astronomical Unit". Celestial Mechanics and Dynamical Astronomy, 103, 365-372. doi: 10.1007/s10569-009-9203-8 